\title{
Association Between Empathy and Burnout Among Emergency Medicine Physicians
}

\author{
Jon A. Wolfshohla, Keegan Bradley ${ }^{\mathrm{a}}$, Charles Bella, Sarah Bell', Caleb Hodges ${ }^{\mathrm{a}}$, \\ Heidi Knowles ${ }^{\mathrm{a}}$, Bharti R. Chaudharia Ryan Kirby ${ }^{\mathrm{a}}$, \\ Jeffrey A. Kline ${ }^{\mathrm{b}}$, Hao Wang ${ }^{\mathrm{a}, \mathrm{c}}$
}

\begin{abstract}
Background: The association between physician self-reported empathy and burnout has been studied in the past with diverse findings. We aimed to determine the association between empathy and burnout among United States emergency medicine (EM) physicians using a novel combination of tools for validation.

Methods: This was a prospective single-center observational study. Data were collected from EM physicians. From December 1, 2018 to January 31, 2019, we used the Jefferson scale of empathy (JSE) to assess physician empathy and the Copenhagen burnout inventory (CBI) to assess burnout. We divided EM physicians into different groups (residents in each year of training, junior/senior attendings). Empathy, burnout scores and their association were analyzed and compared among these groups.
\end{abstract}

Results: A total of 33 attending physicians and 35 EM residents participated in this study. Median self-reported empathy scores were 113 (interquartile range (IQR): 105 - 117) in post-graduate year (PGY)-1, 112 (90 - 115) in PGY-2, 106 (93 - 118) in PGY-3 EM residents, 112 $(105-116)$ in junior and $114(101-125)$ in senior attending physicians. Overall burnout scores were 43 (33 - 50) in PGY-1, 51 (29 - 56) in PGY-2, 43 (42 - 53) in PGY-3 EM residents, 33 (24 - 47) in junior attending and $25(22-53)$ in senior attending physicians separately. The Spearman correlation $(\rho)$ was -0.11 and $\beta$-weight was -0.23 between empathy and patient-related burnout scores.

Conclusion: Self-reported empathy declines over the course of EM residency training and improves after graduation. Overall high burnout occurs among EM residents and improves after graduation. Our analysis showed a weak negative correlation between self-reported empathy and patient-related burnout among EM physicians.

Manuscript submitted May 15, 2019, accepted June 3, 2019

aDepartment of Emergency Medicine, Integrative Emergency Services, John Peter Smith Health Network, 1500 S. Main St., Fort Worth, TX 76104, USA bepartment of Emergency Medicine, Indiana University School of Medicine, 1701 Senae Blvd, Indianapolis, IN 46202, USA

${ }^{\mathrm{c} C o r r e s p o n d i n g ~ A u t h o r: ~ H a o ~ W a n g, ~ D e p a r t m e n t ~ o f ~ E m e r g e n c y ~ M e d i c i n e, ~}$ Integrative Emergency Services, John Peter Smith Health Network, 1500 S. Main St., Fort Worth, TX 76104, USA. Email: hwang01@jpshealth.org

doi: https://doi.org/10.14740/jocmr3878
Keywords: Emergency medicine; Empathy; Burnout

\section{Introduction}

Empathy, in the context of patient care, is defined as the healthcare provider's ability to understand a patient's feelings and thoughts, and effectively communicate that understanding [1]. It directly correlates with patients' healthcare outcomes $[2,3]$. Higher provider empathy leads to fewer medical errors, improved patient satisfaction and reduced incidence of malpractice claims [4-8]. In addition to these important patient-centered outcomes, empathy may also improve provider wellbeing [911]. A growing body of literature suggests that healthcare workers who show greater empathy experience strong emotional intelligence, less stress and lower levels of burnout [10, 12-14].

Physician burnout is characterized by emotional exhaustion, finding less meaning in work, feelings of ineffectiveness and dehumanization of others [15]. Although career burnout is certainly not limited to physicians, it appears to be far more prevalent when compared to the general population [16]. In addition, physician burnout is on the rise and emergency physicians are among those at highest risk [16]. These trends are concerning considering what is at stake - burnout has been associated with increased medical errors, decreased provider efficiency and lower patient satisfaction [17-19]. In recent years, an association between empathy and burnout has been reported in nurses, residents and primary care physicians [20-22]. However, the strength of this relationship seems to largely depend on the variety of definitions of "empathy" and "burnout" used, study sample size and healthcare settings [23].

There are various survey tools used to measure empathy, including Hogan's empathy scale, interpersonal reactivity index (IRI), balanced emotional empathy scale (BEES), and the Jefferson scale of empathy (JSE), to list a few [24-27]. JSE is one of the most common tools to assess physician empathy and has been validated in previous studies $[3,5]$. Notably, the JSE varies among providers depending on medical practice, gender and experience level, but appears to be stable with high test reliability [5]. Physician burnout has also been measured by a variety of tools, such as Maslach burnout inventory (MBI), Hamburg burnout inventory (HBI), Astudillo and Mendinueta burnout questionnaire and Copenhagen burnout inventory (CBI) [28- 
31]. MBI has been used for burnout assessment and accounts for over $80 \%$ of burnout reports in the literature $[15,16]$. MBI measures emotional exhaustion, depersonalization and personal accomplishment domains. However, inconsistencies in definitions of burnout by MBI result in a very wide overall burnout prevalence ranging from $0 \%$ to over $80 \%$ [15]. In the recent years, CBI has been developed in response to perceived limitations of the MBI. Burnout is sometimes conceptualized as a solely work-related exhaustion syndrome, thus measurement tools such as MBI focus on work-related sources of burnout. CBI, on the other hand, broadens the conception of burnout and measures it within three separate domains (personal-, work- and client/patient-related burnout), thus differentiating between different environmental sources and manifestations of this complex phenomenon.

Physician empathy and burnout have become prevalent topics in recent medical literature $[10,22]$. However, there is a paucity of data on the association of empathy and burnout specifically in the field of emergency medicine (EM). Furthermore, we are still uncertain if emergency department (ED) provider empathy is associated with overall provider burnout or more specifically work- or patient- related. A more insightful understanding of this association could lead to improved physician wellness and effective interventions leading to improved patient care. Therefore, we aimed to measure provider empathy by JSE and burnout by CBI to further determine the association between empathy and burnout specifically amongst ED physicians.

\section{Materials and Methods}

\section{Study design and setting}

This was a prospective single-center observational study. This study was conducted in a tertiary referral county hospital. The study hospital is a level-1 trauma center, a comprehensive stroke center and chest pain center. The study hospital ED has an ACGME-sponsored EM residency program with a total of 38 EM residents. Study ED has 53 licensed beds with an annual patient volume of more than 125,000 . This study was approved by local Institutional Review Board. This study was conducted in compliance with the ethical standards of the responsible institution on human subjects as well as with the Helsinki Declaration.

\section{Study participants}

From December 1, 2018 to January 31, 2019, all ED attending physicians and EM residents were included in this study. JSE and CBI were sent to all participants via Survey Monkey. We excluded subjects who: 1) did not complete survey within study period; or 2) completed less than $20 \%$ of study survey questions.

\section{Provider empathy and burnout measurement}

JSE was used for provider empathy measurement. Briefly, we used JSE-Health Professional Version (a revised version of the scale for physicians and health professionals) which includes a 20 -item questionnaire with positively and negatively worded questions. Responses to each of the positive questions is on a seven-point Likert scale ("strongly disagree" = 1 to "strongly agree" $=7$ ). The negative questions are reverse scored using the same seven-point Likert scale ("strongly disagree" $=7$ to "strongly agree" =1). The grand total is summed across all 20 questions for scores ranging from 20 to 140.

$\mathrm{CBI}$, one of the branches of Copenhagen psychosocial questionnaire (COPSOQ), is used to discover stress and burnout in three categories: personal burnout, work-related burnout and patient-related burnout. Briefly, personal burnout is a state of prolonged physical and psychological exhaustion, work-related burnout describes such exhaustion perceived to be related to the person's work, while patient-related burnout more specifically identifies burnout related to provider's work with patients. This is a simplified survey assessment to evaluate provider burnout and further determine whether such burnout relates to providers' personal life, work and/or patients. CBI includes a total of 19-item questionnaires consisting of six questions for personal, seven questions for work-related and six questions for patient-related burnout. These questions include both positively and negatively worded questions. Responses to positive worded questions range from either "always" to "never/almost" or "to very high degree" to "to very low degree" ("always" or "to a very high degree" = 100, "often" or "to a high degree" $=75$, "sometimes" or "somewhat" $=50$, "seldom" or "to a low degree" $=25$ and "never/almost never" or "to a very low degree" $=0$ ). Again, the negative questions are reverse scored. Total score on the scale is the average of the scores on the items.

\section{Study protocol}

We divided our physicians into two groups (resident versus attending physicians). The physicians' basic characteristics including age, gender and race were compared between groups. In addition, three subgroups were further divided from the residents based on training level (e.g. post-graduate year (PGY)-1, PGY-2 and PGY-3). We also further divided attending physicians into two subgroups (e.g. junior versus senior attending physicians). Physicians who recently graduated from EM residency program ( $\leq 5$ years) were placed in the junior attending physician group, whereas those more than 5 years after residency graduation were placed in the senior attending physician group. Empathy, burnout scores and their association were analyzed and compared among different subgroups. Lastly, we categorized empathy scores into low $(<100)$, medium $(100-120)$, high $(121-130)$ and extremely high $(>130)$ score groups, and median burnout scores were also compared among different empathy score groups.

\section{Variables}

We included ED physicians' ages (four categories $(\leq 29,30$ - 
Table 1. Study Participant General Characteristics

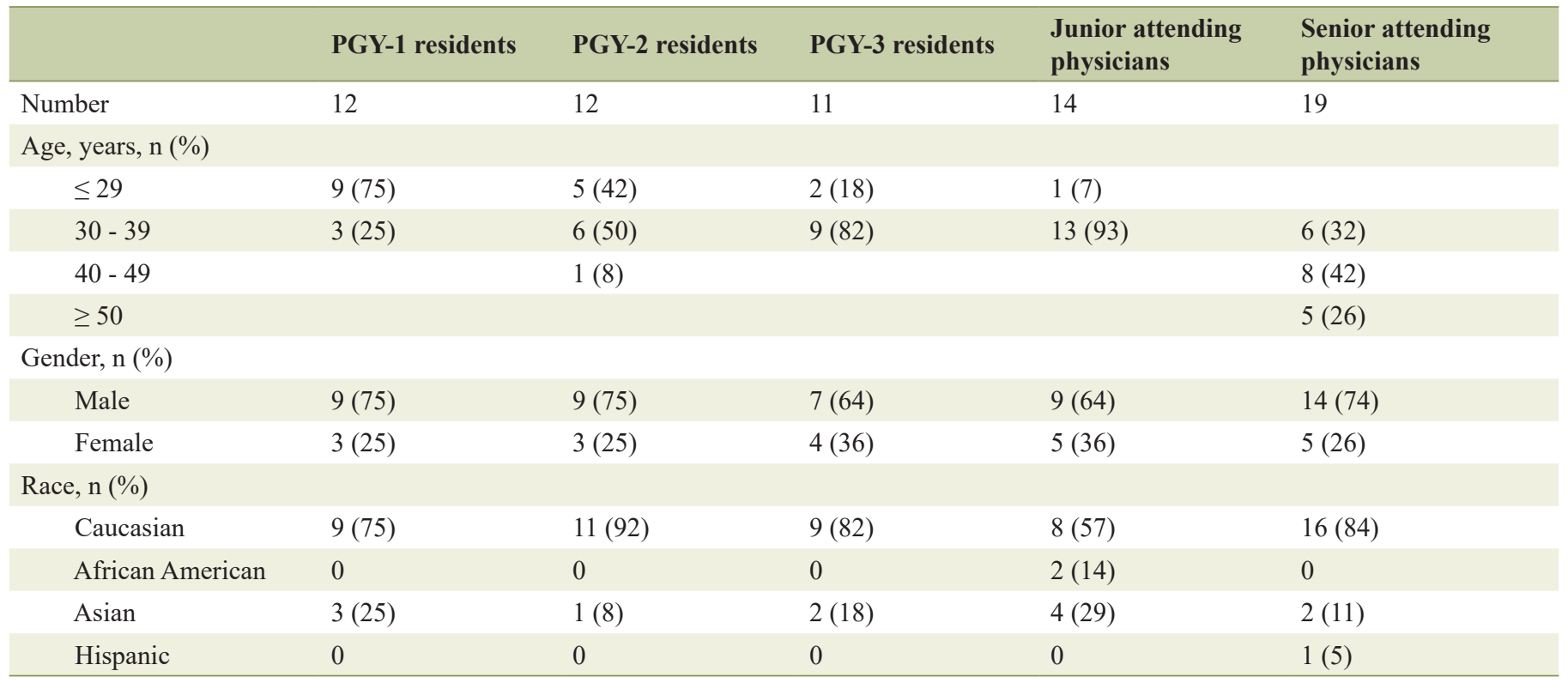

PGY: post-graduate year.

39, 40 - 49, $\geq 50$ )), races (Caucasian, African American and Asian), gender (male versus female), years of training experience (PGY-1, PGY-2 and PGY-3) among EM residents and years of practice experience (junior and senior) among attending physicians.

\section{Data analysis}

We use analysis of variance (ANOVA) to compare continuous data and Chi-square test for categorical data analysis among different groups. Skewness and kurtosis were used to determine data distribution. $\mid$ Skewness $\mid<0.5$ was considered data to be normally distributed, $1>\mid$ skewness $\mid \geq 0.5$ was considered data mildly skewed and $\mid$ skewness $\mid \geq 1$ was considered data highly skewed. Meanwhile, kurtosis $>3$ is also considered data less normally distributed. We use Cronbach's alpha $(\alpha)$ to determine internal consistency of JSE empathy, CBI personal-related, work-related and patient-related burnout separately. An $\alpha>0.8$ is considered good reliability and $\alpha>0.7$ is considered adequate reliability. To determine the association between empathy and burnout, we converted empathy and burnout scores to ordinary ranks instead of using their absolute scores due to mildly skewed data. Furthermore, we subdivided JSE into three subscales including "perspective taking", "compassionate care" and "standing in the patient's shoes" based on previous report in the literature [32]. Correlations were also measured between JSE subscales and three individual CBI domains. Spearman's rho $(\rho)$ test was used to determine such correlations. $|r| \geq 0.5$ indicates strong correlation, $0.5>|r| \geq 0.3$ indicates moderate relationship and $0.3>|\mathrm{r}| \geq 0.1$ indicates a weak relationship. Meanwhile, linear regression was performed to determine the relationship between empathy and burnout with a reported $\beta$ weight. The $\beta$ is a measure of the effect size and is another mean of interpreting correlation with $\beta \geq 0.5$ being strong, $0.5>\beta \geq 0.3$ being moderate and $0.3>\beta \geq 0.1$ being weak. All analyses were performed using Stata v14.0 (College Station, TX, USA).

\section{Results}

A total of 68 ED providers were enrolled in this study, 64 completed the empathy survey and 65 completed the burnout survey. Among 68 ED providers, 35 of which were EM residents, 14 providers were junior attending physicians and 19 were senior attending physicians. The completion rate was 92\% (35/38) among EM residents and 94\% (33/35) in attending physicians. ED providers' general characteristics are shown in Table 1.

Descriptive statistics including mean with standard deviation (SD), median with interquartile range (IQR), skewness, kurtosis and Cronbach's $\alpha$ analysis for the JSE and CBI are reported in Table 2. The JSE mean score was 109 (14) and its median score was 112 (102 - 118). The CBI mean score was 41 (17) and median score was 43 (25 - 53). The skewness was -0.50 for JSE and 0.44 for CBI (Table 2). The kurtosis for the JSE score distribution was 2.77 and for CBI was 3.78, indicating the data are mildly skewed (Table 2). We then performed Cronbach's alpha analysis to determine internal consistency on empathy and burnout surveys. Both surveys showed good reliability with $\alpha>0.8$ (Table 2 ) indicating strong internal consistency among individual providers.

In addition, analysis was performed among subgroups. The median empathy scores decreased with each successive year of resident training. To the contrary, the median empathy scores were higher among junior attendings and further increased among senior attending physicians (Table 3). Personal-related burnout scores seem to have little change among 
Table 2. Descriptive Analysis of the JSE and CBI Scores

\begin{tabular}{llllll}
\hline & JSE & CBI (personal-related) & CBI (work-related) & CBI (patient-related) & CBI (overall) \\
\hline Mean (SD) & $109(14)$ & $45(17)$ & $43(19)$ & $33(19)$ & $41(17)$ \\
Median (IQR) & $112(102-118)$ & $46(33-54)$ & $43(29-54)$ & $33(21-50)$ & $43(25-53)$ \\
Possible range & $20-140$ & $0-100$ & $0-100$ & $0-100$ & $0-100$ \\
Actual range & $74-133$ & $0-100$ & $7.14-100$ & $0-100$ & $2.63-100$ \\
Skewness & -0.50 & 0.26 & 0.40 & 0.55 & 0.44 \\
Kurtosis & 2.77 & 3.54 & 3.07 & 3.71 & 3.78 \\
Cronbach's $\alpha$ & 0.86 & 0.89 & 0.88 & 0.90 & 0.91 \\
\hline
\end{tabular}

JSE: Jefferson scale of empathy; CBI: Copenhagen burnout inventory; SD: standard deviation; IQR: interquartile range.

residents. However, work-related and patient-related burnout scores were higher among PGY-2 EM residents, resulting in higher overall burnout scores among this cohort. Further dividing empathy score into four categories (low, medium, high and extremely high) shows the trend of decreased burnout scores with increased empathy (Table 3 ). In addition, male providers tend to have lower empathy scores (Table $3, \mathrm{P}=0.26$ ) but higher burnout scores compared to females (Table 3, $\mathrm{P}=0.74$ ).

When correlation was performed, only patient-related burnout showed weak-to-moderate correlation with providers' empathy. More specifically, patient-related burnout showed weak-to-moderate correlation with both "compassionate care" and "standing in the patient's shoes" subscales of JSE. Such findings were very similar regardless of different correlation analyses performed (Table 4).

\section{Discussion}

Using JSE for self-assessed empathy measurement and CBI for burnout assessment among ED physicians, our results show the trend of increased physician self-assessed empathy score with decreased burnout levels and further demonstrate a weak correlation between providers' empathy and their patient-related burnout. No such correlation was seen between provider empathy and either personal or work-related burnout. Given that the JSE empathy tool for assessing physician empathy is specific to patient care [1], it is not surprising that such empathy correlates with patient-related burnout. Our results are consistent with other studies reporting the association between provider empathy and burnout $[10,33]$. Apart from the investigation of our primary outcomes, we also found trends of increased empathy and decreased burnout after physicians graduate from residency and further as they become senior attendings, which have not previously been reported extensively.

Numerous studies have reported that a high level of physician burnout correlates with low empathy scores to varying degrees $[10,20,33]$. However, the majority of these investigations have measured physician burnout using MBI and, to our knowledge, burnout's association with empathy has not been previously studied among US emergency physicians [10, 22, 33]. Using JSE and CBI, our study observed a weak association between empathy and burnout in the domain of patient-related burnout among US emergency physicians. The weaker cor-

Table 3. A Comparison of Empathy and Burnout Score Among Different Provider Groups

\begin{tabular}{|c|c|c|c|c|c|}
\hline & Empathy & $\begin{array}{l}\text { Personal-related } \\
\text { burnout }\end{array}$ & $\begin{array}{l}\text { Work-related } \\
\text { burnout }\end{array}$ & $\begin{array}{l}\text { Patient-related } \\
\text { burnout }\end{array}$ & Overall burnout \\
\hline PGY-1 residents & $113(105-117)$ & $50(44-54)$ & $48(38-55)$ & $29(23-42)$ & $43(33-50)$ \\
\hline PGY-3 residents & $106(93-118)$ & $50(46-58)$ & $43(39-57)$ & $38(38-54)$ & $43(42-53)$ \\
\hline Junior attending & $112(105-116)$ & $42(33-58)$ & $39(32-50)$ & $25(21-33)$ & $33(24-47)$ \\
\hline Low-score & $90(81-92)$ & $38(29-50)$ & $43(32-50)$ & $33(8-54)$ & $43(25-50)$ \\
\hline Medium-score & $112(106-116)$ & $50(38-58)$ & $43(36-57)$ & $35(21-50)$ & $43(32-54)$ \\
\hline High-score & $125(123-127)$ & $38(29-50)$ & $25(16-55)$ & $23(19-58)$ & $24(23-55)$ \\
\hline Extremely high-score & $131(131-133)$ & $21(17-79)$ & $21(14-79)$ & $8(8-42)$ & $17(13-67)$ \\
\hline
\end{tabular}

PGY: post-graduate year. 
Table 4. Relationship Between Empathy and Burnout Among ED Providers

\begin{tabular}{lllll}
\hline & Overall burnout & Personal-related burnout & Work-related burnout & Patient-related burnout \\
\hline Overall empathy & $\mathrm{r}=-0.03$ & $\mathrm{r}=0.04$ & $\mathrm{r}=-0.05$ & $\mathrm{r}=-0.11$ \\
& $\beta=-0.02$ & $\beta=0.26$ & $\beta=-0.05$ & $\beta=-0.23$ \\
Perspective taking & $\mathrm{r}=-0.02$ & $\mathrm{r}=0.05$ & $\mathrm{r}=-0.05$ & $\mathrm{r}=-0.03$ \\
& $\beta=0.00$ & $\beta=0.25$ & $\beta=-0.22$ & $\beta=-0.02$ \\
Compassionate care & $\mathrm{r}=-0.01$ & $\mathrm{r}=0.03$ & $\mathrm{r}=-0.03$ & $\mathrm{r}=-0.10$ \\
& $\beta=-0.02$ & $\beta=0.20$ & $\beta=-0.08$ & $\beta=-0.31$ \\
Standing in the patients' shoes & $\mathrm{r}=-0.08$ & $\mathrm{r}=-0.05$ & $\mathrm{r}=-0.08$ & $\mathrm{r}=-0.15$ \\
& $\beta=-0.07$ & $\beta=0.13$ & $\beta=-0.09$ & $\beta=-0.30$ \\
\hline
\end{tabular}

ED: emergency department.

relation between empathy and burnout observed in our study may be attributable to multiple factors. The difference could be: 1) a unique study population, physicians practicing EM in a high volume US level-1 trauma center; 2) different risks affecting empathy and burnout differently including fatigue, psychological conditions, etc. [34]; 3) an intrinsic property of the burnout assessment used in our study. Burnout determined by $\mathrm{CBI}$ tends to be more environmental or conditional (e.g. workor patient-related), whereas, burnout determined by MBI tends to be more general and personal (e.g. depersonalization, personal accomplishment) $[15,35]$; and 4) more specifically, MBI gauges "depersonalization" through questions such as "I treated patients as if they were impersonal objects" and thus may be more likely to negatively correlate with empathy, or a care provider's ability to understand a patient's feelings, than CBI [36]. Although our findings show some level of association between empathy and burnout when using a novel combination of assessment tools in a unique physician population, a multicenter study is warranted to further determine the direct link between empathy and burnout.

Other findings from this study correlate well with previous studies. For instance, female providers empathy scores were higher than males [5, 37], and empathy scores decreased among residents as they progressed in their training $[5,38]$. Our findings of increased empathy among senior attending physicians have also been previously described [5]. We found residents and junior attendings had relatively higher burnout scores than senior attendings. This finding seems consistent across the literature regardless of the burnout measurement tool used $[39,40]$. These trends seen in our study and others indicate some generalizability of empathy and burnout characteristics among physicians.

However, other trends observed in our study are still controversial in the literature. Some studies have shown higher burnout among female physicians when compared to their male counterparts [41, 42], while others reported no significant difference between genders $[40,43]$. Our study showed relatively higher self-reported burnout in males than females but was unable to reach significant level due to limited sample size, thus warranting further investigations in the future. We found personal-related burnout at comparable levels among all residents and junior attendings, which could be due to the similar ages represented in these groups often facing similar personal/family events (such as family stress, financial burden, unstable job security and personal career building, etc.) [4446]. In addition, PGY-2 residents had higher burnout scores than other groups, especially work-related burnout. We assume that the second EM training year may demonstrate higher stress since residents experience a steep learning-curve during this year and begin taking more responsibility for department leadership and career development.

Since both low empathy and high burnout are related to poor patient care outcomes $[3,18]$, determining the association between empathy and burnout among ED providers may lead to better emergency care. Furthermore, a better understanding of empathy and burnout could help develop effective physician wellness programs. Our future research will be focused on changes in ED provider empathy and burnout with different interventions.

Our study has its limitations. First, this is a single centered trial with a small sample size, and we are unable to reach statistically significant differences among different groups when analyzed. Therefore, we can only report the trends of such associations between empathy and burnout in ED physicians which need future validation in a large-scale sample size study. Second, we only used JSE and CBI tools to assess provider empathy and burnout, respectively, without exploring use of other tools for comparison. In addition, the study survey was only performed once and was not repeated to assess test-retest reliability. Third, our study subjects did not include physician assistants or nurse practitioners in the groups, nor were physicians surveyed during holiday times when swings in both empathy and burnout might be noted. In addition, it is likely that empathy and burnout levels are affected multi-factorially, and we were unable to analyze for all potential confounders. Therefore, a large-scale, multicenter prospective study would allow us to further assess the relationship of empathy and burnout in the ED.

\section{Conclusion}

Using the JSE and CBI to assess empathy and burnout respectively, our study found a weak negative correlation between emergency physician empathy and patient-related burnout. There was no correlation between empathy and overall burnout. We found higher self-assessed physician empathy and lower burnout scores among attending physicians with 
increased practice experience, especially senior ones, when compared to residents.

\section{Acknowledgments}

None.

\section{Financial Disclosure}

None.

\section{Conflict of Interest}

None.

\section{Informed Consent}

Approved by local Institutional Review Board with waived written informed consent.

\section{Author Contributions}

HW, JW, HK and JAK conceived the study and designed the protocol. $\mathrm{HW}, \mathrm{JW}, \mathrm{KB}, \mathrm{HK}, \mathrm{BC}$ and $\mathrm{RK}$ performed the literature search, and review. JW, $\mathrm{CB}, \mathrm{SB}$ and $\mathrm{CH}$ preformed data collection and coding. HW, JW, HK and JAK performed the data interpretation and analysis. HW, JW and KB drafted the initial article and all other authors provided critical revisions and contributed substantially to this study. All authors approved the final version of the manuscript to be published. All authors agreed to be accountable for all aspects of the work in ensuring the questions related to the accuracy or integrity of any part of the work. HW takes final responsibility for the paper.

\section{References}

1. Hojat M, Gonnella JS, Nasca TJ, Mangione S, Vergare M, Magee M. Physician empathy: definition, components, measurement, and relationship to gender and specialty. Am J Psychiatry. 2002;159(9):1563-1569.

2. Del Canale S, Louis DZ, Maio V, Wang X, Rossi G, Hojat M, Gonnella JS. The relationship between physician empathy and disease complications: an empirical study of primary care physicians and their diabetic patients in Parma, Italy. Acad Med. 2012;87(9):1243-1249.

3. Hojat M, Louis DZ, Markham FW, Wender R, Rabinowitz C, Gonnella JS. Physicians' empathy and clinical outcomes for diabetic patients. Acad Med. 2011;86(3):359364.

4. Neumann M, Scheffer C, Tauschel D, Lutz G, Wirtz M, Edelhauser F. Physician empathy: definition, outcome- relevance and its measurement in patient care and medical education. GMS Z Med Ausbild. 2012;29(1):Doc11.

5. Wang H, Kline JA, Jackson BE, Laureano-Phillips J, Robinson RD, Cowden CD, d'Etienne JP, et al. Association between emergency physician self-reported empathy and patient satisfaction. PLoS One. 2018;13(9):e0204113.

6. Ogle J, Bushnell JA, Caputi P. Empathy is related to clinical competence in medical care. Med Educ. 2013;47(8):824-831.

7. Sonis JD, Aaronson EL, Lee RY, Philpotts LL, White BA. Emergency department patient experience: a systematic review of the literature. J Patient Exp. 2018;5(2):101106.

8. Smith DD, Kellar J, Walters EL, Reibling ET, Phan T, Green SM. Does emergency physician empathy reduce thoughts of litigation? A randomised trial. Emerg Med J. 2016;33(8):548-552.

9. Dagnone JD. Commentary: The physician as person framework: How human nature impacts empathy, depression, burnout, and the practice of medicine. Can Med Educ J. 2017;8(4):e97-e98.

10. Yuguero O, Forne C, Esquerda M, Pifarre J, Abadias MJ, Vinas J. Empathy and burnout of emergency professionals of a health region: A cross-sectional study. Medicine (Baltimore). 2017;96(37):e8030.

11. Dasan S, Gohil P, Cornelius V, Taylor C. Prevalence, causes and consequences of compassion satisfaction and compassion fatigue in emergency care: a mixedmethods study of UK NHS Consultants. Emerg Med J. 2015;32(8):588-594.

12. Bertram K, Randazzo J, Alabi N, Levenson J, Doucette JT, Barbosa P. Strong correlations between empathy, emotional intelligence, and personality traits among podiatric medical students: A cross-sectional study. Educ Health (Abingdon). 2016;29(3):186-194.

13. Park KH, Kim DH, Kim SK, Yi YH, Jeong JH, Chae J, Hwang J, et al. The relationships between empathy, stress and social support among medical students. Int J Med Educ. 2015;6:103-108.

14. Crowe RP, Bower JK, Cash RE, Panchal AR, Rodriguez SA, Olivo-Marston SE. Association of burnout with workforce-reducing factors among EMS professionals. Prehosp Emerg Care. 2018;22(2):229-236.

15. Rotenstein LS, Torre M, Ramos MA, Rosales RC, Guille C, Sen S, Mata DA. Prevalence of burnout among physicians: a systematic review. JAMA. 2018;320(11):11311150.

16. Shanafelt TD, Hasan O, Dyrbye LN, Sinsky C, Satele D, Sloan J, West CP. Changes in burnout and satisfaction with work-life balance in physicians and the General US working population between 2011 and 2014. Mayo Clin Proc. 2015;90(12):1600-1613.

17. Shanafelt TD, Mungo M, Schmitgen J, Storz KA, Reeves D, Hayes SN, Sloan JA, et al. Longitudinal study evaluating the association between physician burnout and changes in professional work effort. Mayo Clin Proc. 2016;91(4):422-431.

18. Dewa CS, Loong D, Bonato S, Trojanowski L. The relationship between physician burnout and quality of health- 
care in terms of safety and acceptability: a systematic review. BMJ Open. 2017;7(6):e015141.

19. Halbesleben JR, Rathert C. Linking physician burnout and patient outcomes: exploring the dyadic relationship between physicians and patients. Health Care Manage Rev. 2008;33(1):29-39.

20. Yuguero O, Ramon MJ, Esquerda M, Vivanco L, SolerGonzalez J. Association between low empathy and high burnout among primary care physicians and nurses in Lleida, Spain. Eur J Gen Pract. 2017;23(1):4-10.

21. Yuguero O, Melnick ER, Marsal JR, Esquerda M, SolerGonzalez J. Cross-sectional study of the association between healthcare professionals' empathy and burnout and the number of annual primary care visits per patient under their care in Spain. BMJ Open. 2018;8(7):e020949.

22. Park C, Lee YJ, Hong M, Jung CH, Synn Y, Kwack YS, Ryu JS, et al. A Multicenter Study Investigating Empathy and Burnout Characteristics in Medical Residents with Various Specialties. J Korean Med Sci. 2016;31(4):590597.

23. Williams B, Lau R, Thornton E, Olney LS. The relationship between empathy and burnout - lessons for paramedics: a scoping review. Psychol Res Behav Manag. 2017;10:329-337.

24. Froman RD, Peloquin SM. Rethinking the use of the Hogan Empathy Scale: a critical psychometric analysis. Am J Occup Ther. 2001;55(5):566-572.

25. Bonfils KA, Lysaker PH, Minor KS, Salyers MP. Empathy in schizophrenia: A meta-analysis of the Interpersonal Reactivity Index. Psychiatry Res. 2017;249:293-303.

26. Balconi M, Canavesio Y. Emotional contagion and trait empathy in prosocial behavior in young people: the contribution of autonomic (facial feedback) and balanced emotional empathy scale (BEES) measures. J Clin Exp Neuropsychol. 2013;35(1):41-48.

27. Kane GC, Gotto JL, Mangione S, West S, Hojat M. Jefferson Scale of patient's perceptions of physician empathy: preliminary psychometric data. Croat Med J. 2007;48(1):81-86.

28. Maslach C, Jackson SE, Leiter MP. Maslach Burnout Inventory Manual, 4th edn. Menlo Park, CA: Mind Garden Inc.; 2016.

29. Kristensen TS, Borritz M, Villadsen E, Christensen KB. The Copenhagen burnout inventory: A new tool for the assessment of burnout. Work Stress. 2005;19:192-207.

30. Burisch M. Approaches to personality inventory construction: a comparison of merits. Am Psychol. 1984;39:214227.

31. Astudillo W, Mendinueta C. Exhaustion syndrome in palliative care. Support Care Cancer. 1996;4(6):408-415.

32. Hojat M, DeSantis J, Shannon SC, Mortensen LH, Speicher MR, Bragan L, LaNoue M, et al. The Jefferson Scale of Empathy: a nationwide study of measurement properties, underlying components, latent variable structure, and national norms in medical students. Adv Health Sci
Educ Theory Pract. 2018;23(5):899-920.

33. Lee PT, Loh J, Sng G, Tung J, Yeo KK. Empathy and burnout: a study on residents from a Singapore institution. Singapore Med J. 2018;59(1):50-54.

34. Picard J, Catu-Pinault A, Boujut E, Botella M, Jaury P, Zenasni F. Burnout, empathy and their relationships: a qualitative study with residents in General Medicine. Psychol Health Med. 2016;21(3):354-361.

35. Cheng Y, Chen IS, Chen CJ, Burr H, Hasselhorn HM. The influence of age on the distribution of self-rated health, burnout and their associations with psychosocial work conditions. J Psychosom Res. 2013;74(3):213-220.

36. Hojat M, Vergare M, Isenberg G, Cohen M, Spandorfer J. Underlying construct of empathy, optimism, and burnout in medical students. Int J Med Educ. 2015;6:12-16.

37. Hojat M, Gonnella JS, Nasca TJ, Mangione S, Veloksi JJ, Magee M. The Jefferson Scale of Physician Empathy: further psychometric data and differences by gender and specialty at item level. Acad Med. 2002;77(10 Suppl):S58-60.

38. Hojat M, Mangione S, Nasca TJ, Rattner S, Erdmann JB, Gonnella JS, Magee M. An empirical study of decline in empathy in medical school. Med Educ. 2004;38(9):934941.

39. Dyrbye LN, West CP, Satele D, Boone S, Tan L, Sloan J, Shanafelt TD. Burnout among U.S. medical students, residents, and early career physicians relative to the general U.S. population. Acad Med. 2014;89(3):443-451.

40. Starmer AJ, Frintner MP, Freed GL. Work-Life Balance, Burnout, and Satisfaction of Early Career Pediatricians. Pediatrics. 2016;137(4):e20153183.

41. Shenoi AN, Kalyanaraman M, Pillai A, Raghava PS, Day S. Burnout and Psychological Distress Among Pediatric Critical Care Physicians in the United States. Crit Care Med. 2018;46(1):116-122.

42. Amoafo E, Hanbali N, Patel A, Singh P. What are the significant factors associated with burnout in doctors? Occup Med (Lond). 2015;65(2):117-121.

43. Schooley B, Hikmet N, Tarcan M, Yorgancioglu G. Comparing burnout across emergency physicians, nurses, technicians, and health information technicians working for the same organization. Medicine (Baltimore). 2016;95(10):e2856.

44. Kuhn G, Goldberg R, Compton S. Tolerance for uncertainty, burnout, and satisfaction with the career of emergency medicine. Ann Emerg Med. 2009;54(1):106-113 e106.

45. Jalili M, Sadeghipour Roodsari G, Bassir Nia A. Burnout and Associated Factors among Iranian Emergency Medicine Practitioners. Iran J Public Health. 2013;42(9):10341042.

46. Hunsaker S, Chen HC, Maughan D, Heaston S. Factors that influence the development of compassion fatigue, burnout, and compassion satisfaction in emergency department nurses. J Nurs Scholarsh. 2015;47(2):186-194. 